\title{
STUDY OF THE COMPOSITE SUPERNOVA REMNANT MSH 11-62
}

\author{
Ilana M. Harrus ${ }^{1,2,3}$, John P. Hughes ${ }^{1,4}$ and Patrick O. Slane ${ }^{1,5}$
}

Received October 1997; $\quad$ accepted January 1998

Accepted for publication in The Astrophysical Journal

\footnotetext{
${ }^{1}$ Harvard-Smithsonian Center for Astrophysics, 60 Garden Street, Cambridge, MA 021381516

2 Department of Physics, Columbia University, 550 West $120^{\text {th }}$ Street, New York, NY 10027

3 imh@head-cfa.harvard.edu

${ }^{4}$ Department of Physics and Astronomy, Rutgers University, 136 Frelinghuysen Road, Piscataway, NJ 08854-8019; jph@physics.rutgers.edu

5 slane@cfa.harvard.edu
} 


\begin{abstract}
We present the analysis of the X-ray data collected during an observation of the supernova remnant (SNR) MSH 11-62 by the Advanced Satellite for Cosmology and Astrophysics (ASCA). We show that MSH 11-62 is a composite remnant whose X-ray emission comes from two distinct contributions. Nonthermal, synchrotron emission, localized to a region of radius $\sim 3^{\prime}$ (consistent with a point source) dominates the total flux above $2 \mathrm{keV}$. A second contribution comes from a thermal component, extended up to a radius of $\sim 6^{\prime}$ and detected only at energies below $2 \mathrm{keV}$. The spatial and spectral analysis imply the presence of a neutron star losing energy at a rate of about $\dot{E} \sim 10^{36}-10^{37}$ ergs $\mathrm{s}^{-1}$. No pulsed emission is detected and we set a limit on the pulsed fraction of $\sim 10 \%$. This is consistent with the lack of a radio pulsar in the remnant, which may indicate that the pulsed emission from the rapidly rotating compact object that should be powering the synchrotron nebula is beamed and our viewing direction is unfavorable. In either event, the central neutron star deposits much of its spin-down energy into the surrounding synchrotron nebula where, through direct imaging with broadband satellites such as $A S C A$, it is possible to study the energetics and evolution of the compact remnant.
\end{abstract}

Subject headings: radiation mechanisms: non-thermal — supernova remnants: individual: MSH 11-62 - X-rays: ISM 


\section{Introduction}

In the standard scenario of the core collapse of a massive star, a shock wave propagates ahead of the ejecta through the interstellar medium (ISM) forming a supernova remnant (SNR). The X-ray emission comes from a thin shell of interstellar material swept-up by the supernova blast wave and the spectrum shows thermal features indicative of a high-temperature plasma. There exists, however, a class of SNRs which does not follow this standard picture and shows, instead, a centrally peaked X-ray morphology with a nonthermal spectrum. The most famous example of such a remnant is the Crab Nebula in which the X-ray (and radio) flux is synchrotron radiation produced by relativistic electrons and magnetic field all powered by the spin-down energy loss of the compact object, a neutron star, created in the explosion. This nonthermal radiation is the signature of the compact object and provides proof that the remnant was produced by a Type Ib/c or Type II SN (i.e., the core collapse of a massive star). In the case of the Crab Nebula, the $33 \mathrm{~ms}$ pulsar has been detected at all wavelengths including the X-ray band. However, it is often the case that the pulsed emission itself escapes detection (an effect which may be due to beaming). X-ray studies provide another tool to confirm the presence of a compact object by detecting the associated synchrotron nebula or plerion. This method does not suffer

from the beaming effect bias and gives us direct information on the energetics, specifically the current energy output, of the central powering object. With the advance of the high sensitivity broadband X-ray imaging and spectral instruments onboard $A S C A$, the study of this class of objects has made great progress.

$A S C A$ has made it possible to unambiguously identify and quantify the nonthermal emission from synchrotron nebulae and to separate it from the thermal emission seen from shock-heated SNR gas. The SNRs for which this distinction has been made have emerged recently as a growing class. The currently unique imaging capabilities of $A S C A$ at energies above $\simeq 4 \mathrm{keV}$ where the contribution from the thermal emission is negligible, has allowed 
us to directly image the contribution from the synchrotron emission coming from the (often undetected) pulsar. ASCA studies of several SNRs have either confirmed radio data that suggested the presence of a synchrotron nebula (e.g., Kes 75, Blanton \& Helfand 1996; and G11.2-0.3, Vasisht et al. 1996) or directly identified possible new members of this class (e.g., W44, Harrus, Hughes \& Helfand 1996; CTA-1, Slane et al. 1997; and MSH 15-52, Tamura et al. 1996). It is now clear that MSH 11-62 (G291.0-0.1) is also a composite supernova remnant.

Radio observations of MSH 11-62 reveal a centrally-brightened morphology with a spectral index of $\alpha=0.29 \pm 0.05$ and strong linear polarization (Roger et al. 1986). Prior to our $A S C A$ observation, very little was known about this SNR in the $\mathrm{X}$-ray band. It was observed by the Einstein Imaging Proportional Counter (IPC), but these data were insufficient to distinguish between a thermal and a nonthermal interpretation (Wilson 1986). No on-axis pointing of the object was made with the ROSAT Position Sensitive Proportional Counter although an observation with the High Resolution Imager was recently carried out (but the data have not yet been released to the public archive).

Presented here are the results and interpretations of our $A S C A$ X-ray data on the SNR MSH 11-62. We present in $\S 2$ our data extraction methods and spatial, spectral, and timing analyses. In $\S 3$ we discuss the implications of our analysis on the distance to MSH 11-62, its evolutionary state, and the association of the remnant with an unidentified $\gamma$-ray source. We interpret the nonthermal emission as having a synchrotron origin and derive estimates of the mean magnetic field in the synchrotron nebula that compare well with estimates of the thermal pressure in the remnant. Then we tie together the various observational results into two evolutionary scenarios, both of which appear to describe the remnant equally well. Further observational tests that could help distinguish between these scenarios are discussed briefly. The final section of the article is a summary of our principal conclusions. 


\section{Data Reduction and Analysis}

An $A S C A$ observation of MSH 11-62 was carried out on 7 February, 1996, toward the nominal position of the remnant. The event files were cleaned according to the standard prescription as follows: for the Gas Imaging Spectrometer (GIS) detectors, we rejected events recorded when the satellite was traversing regions of low magnetic rigidity $(<6)$ (where the flux of high energy charged particles, and consequently the X-ray background rate, tends to be greater) or when the Earth elevation angle was below $10^{\circ}$. In addition, we also removed the internal background due to the calibration source at the edge of the detector and discarded data located on the external bright ring of the detector. The screening criteria for the Solid-State Imaging Spectrometer (SIS) data were almost identical to those used for the GIS: minimum Earth elevation angle of $10^{\circ}$ and a minimum rigidity of 6 . Because of the possibility for contamination in the SIS of fluorescence lines of oxygen from the Earth's atmosphere, data selection was also done on the angle to the limb of the sunlit Earth, and only data above $40^{\circ}\left(20^{\circ}\right)$ for the SIS0 (SIS1) were retained. Finally, only events with CCD grades $0,2,3$, or 4 were used in further analysis. The various grades characterize the shape of the event as recorded by the CCD. Our selection is the standard recommended set which represents a compromise between the rejection of background (i.e., charged particle events) and real X-ray photons.

Our ASCA observation was planned to study primarily the spectral nature of the SNR. In particular, we retained GIS rise-time information in order to pursue accurate spectral analysis and only assigned two extra bits (from compressing the pulse height spectrum the spectrum is compressed for data analysis in any case) to timing data. This gave us, in the GIS, a time resolution of $0.125 \mathrm{sec}$ in medium-bit-rate mode and $0.015625 \mathrm{sec}$ in high-bit-rate mode. 


\subsection{Spatial Analysis}

We generated exposure-corrected, background-subtracted images of the GIS and SIS data in selected spectral bands. The background was determined from the weighted average of several nominally blank fields from high Galactic latitude observations with data selection criteria matched to those used for the SNR data. Exposure maps were generated from the off-axis effective-area calibrations, weighted by the appropriate observation time. Events from regions of the merged exposure map with less than $10 \%$ of the maximum exposure were ignored. Merged images of the source data, background, and exposure were smoothed with a Gaussian of standard deviation, $\sigma=45^{\prime \prime}$. We subtracted smoothed background maps from the data maps and divided by the corresponding exposure map.

Fig. 1 shows the strikingly different appearance of MSH 11-62 in the soft, medium, and hard X-ray bands as observed by the GIS and the SIS. At energies below 2 keV (top panels in Fig. 1), the emission is extended, covering a region with a diameter of somewhat more than $10^{\prime}$. At higher energies, both between $2 \mathrm{keV}$ and $4 \mathrm{keV}$ (medium panels) and above $4 \mathrm{keV}$ (bottom panels), the extended emission which fills the radio shell has disappeared and only centrally concentrated emission appears. Fig. 2 shows the radio contours from the Molonglo Observatory Synthesis Telescope (MOST) (Whiteoak \& Green 1996) superimposed on the same X-ray images as displayed in Fig. 1. There is a clear correlation between the location of the maximum in radio and the center of the hard X-ray emission. Furthermore, the total extent of the radio SNR agrees fairly well with that of the soft X-ray contours and we estimate the angular radius of the remnant based on the outermost radio contours in Fig. 2 to be $6 ! 4$.

In Fig. 3 we show the variation of an X-ray hardness ratio (which we define to be the number of counts between 2 and $10 \mathrm{keV}$ divided by the number of counts between 0.5 and $2 \mathrm{keV}$ ) as a function of radius. The ratio decreases significantly with radius (which is opposite to the sense of instrumental effects) and confirms that the central emission 
is considerably harder than the emission from the edge. We show below through our spectral analysis that the central hard emission is dominated by a nonthermal, power-law contribution. The size of the hard X-ray source is consistent with an unresolved source $\left(<3^{\prime}\right.$ radius in both SIS and GIS) and its location is $\left(11^{\mathrm{h}} 11^{\mathrm{m}} 48.5^{\mathrm{s}} \pm 1.0^{\mathrm{s}},-60^{\circ} 39^{\prime} 20^{\prime \prime} \pm 15^{\prime \prime}\right)$ in the GIS and $\left(11^{\mathrm{h}} 11^{\mathrm{m}} 48.7^{\mathrm{s}} \pm 1.0^{\mathrm{s}},-60^{\circ} 40^{\prime} 00^{\prime \prime} \pm 15^{\prime \prime}\right)$ in the SIS. This position is consistent with the highly polarized, flat spectrum region at the center of the radio image of MSH 11-62. We estimate the angular size of this region to be 2.5 in radius, where, to account for the significant elongation of the nebula, we have taken the geometric mean of the semi-major and semi-minor axis lengths of the region defined by the third lowest radio contour in Fig. 2.

Above $2.0 \mathrm{keV}$, the GIS and SIS images reveal a hard extended source located at the northeastern edge of the field of view (at approximate position $11^{\mathrm{h}} 13^{\mathrm{m}} 40^{\mathrm{s}},-60^{\circ} 33^{\prime} 30^{\prime \prime}$ ) that is absent at low energies. This source is likely to be the ghost image of the nearby bright X-ray binary Cen X-3 which is located about $1^{\circ}$ east of MSH 11-62. Ghost images are created when X-rays from a source outside the field of view are scattered off only one of the focusing mirror elements. As Fig. 1 shows, this spurious emission is isolated from the center of the field of view where MSH 11-62 lies and, thus, has no impact on our analysis.

\subsection{Spectral Analysis}

We extracted X-ray spectra from circular regions centered at $11^{\mathrm{h}} 11^{\mathrm{m}} 51.49^{\mathrm{s}}$, $-60^{\circ} 39^{\prime} 11.74^{\prime \prime}$ (J2000), using a radius of $5^{\prime}$ in the GIS and, due to its smaller field of view, only $4^{\prime}$ in the SIS. After the standard data selection criteria were applied and within the regions described above, there were 4760 (4949) events in GIS2 (GIS3) and 4726 (4182) events in SIS0 (SIS1). We first fitted the SIS and GIS separately and then jointly. The extended nature of the source and the contamination by the ghost image of Cen X-3 made it impossible to extract a sufficient amount of background data from a blank part of the field, especially for the SIS analysis. For background, therefore, we used the high Galactic 
latitude blank fields available from the $H E A S A R C$, after establishing with the GIS data that the high-energy contamination from the Galactic plane was negligible.

To take into account the high-energy spectrum as well as the extended soft component present below $2 \mathrm{keV}$, we modeled the data with a mixture of nonthermal (i.e., power-law) and thermal components. For the thermal emission we used the Raymond \& Smith (1977) thermal plasma model with cosmic abundances (Anders \& Grevesse 1989). Absorption along the line of sight was taken into account with an equivalent column density of hydrogen, $N_{\mathrm{H}}$, using the cross-sections and abundances from Morrison \& McCammon (1983). A purely nonthermal model failed to take into account the low energy part of the spectrum, while a single thermal model failed to account for the high energy part. Considerably better fits in each case were obtained by including an additional thermal component. However, the exclusively thermal model required an unseemly high second temperature $(\sim 12 \mathrm{keV})$ to account for the high-energy emission. The best overall fit $\left(\chi^{2}=671.9, \chi_{\mathrm{r}}^{2} \sim 2.0\right)$ to the joint GIS and SIS spectral data was obtained for a model including both thermal and nonthermal components. The power-law component has a photon index of $2.0_{-0.3}^{+0.1}$ (the errors quoted here take into account the different analyses carried out - detectors fitted separately, simultaneously, and combined with their respective response file - as well as the standard $1 \sigma$ statistical error). Each pair of nominally identical detectors (GIS and SIS) produced consistent normalizations and so they were linked in the remaining fits. We find the flux density of the power-law at $1 \mathrm{keV}$ in the GIS to be $F_{1 \mathrm{keV}}=(1.26 \pm 0.13) \times 10^{-3}$ photons $\mathrm{cm}^{-2} \mathrm{~s}^{-1} \mathrm{keV}^{-1}$ and $F_{1 \mathrm{keV}}=(1.0 \pm 0.1) \times 10^{-3}$ photons $\mathrm{cm}^{-2} \mathrm{~s}^{-1} \mathrm{keV}^{-1}$ in the SIS. (N.B., the GIS and SIS normalizations differ because the X-ray flux that is lost in the gaps between the CCD chips in the SIS is not accounted for in the effective area used to model these data.) The thermal component has a temperature $k T=0.80 \pm 0.05 \mathrm{keV}$ and an emission measure $\left(n_{\mathrm{H}} n_{e} V / 4 \pi D^{2}\right)$ of $\left(1.9_{-0.5}^{+1.0}\right) \times 10^{11} \mathrm{~cm}^{-5}$ from the GIS and $\left(8.6_{-2.8}^{+5.7}\right) \times 10^{10}$ $\mathrm{cm}^{-5}$ from the SIS.

The column density toward the source is $N_{\mathrm{H}}=(6 \pm 1) \times 10^{21}$ atoms $\mathrm{cm}^{-2}$, consistent 
with the previously derived value of $4 \times 10^{21}$ atoms $\mathrm{cm}^{-2}$ (Wilson 1986). The unabsorbed flux from this thermal component between 0.1 and $2.0 \mathrm{keV}$, computed with the GIS normalization because of its more complete coverage of the source, is $\left(6.3_{-2}^{+4}\right) \times 10^{-12}$ $\operatorname{ergs} \mathrm{cm}^{-2} \mathrm{~s}^{-1}$ contributing $51 \%$ of the total flux in this energy range, while it is only $\left(2.4_{-0.9}^{+1.8}\right) \times 10^{-13} \operatorname{ergs~} \mathrm{cm}^{-2} \mathrm{~s}^{-1}$ above $2.0 \mathrm{keV}$, less than $7 \%$ of the total flux of $\left(3.5_{-0.8}^{+1.1}\right) \times 10^{-12}$ $\operatorname{ergs} \mathrm{cm}^{-2} \mathrm{~s}^{-1}$ from the remnant.

Fig. 4 shows the data and the best-fit power-law plus thermal model. The thermal component is plotted separately in the insert to the figure. Table 1 gives numerical results from the spectral analysis. The residuals in Fig. 4 show the presence of an additional line emission that is not properly taken into account in the standard plasma model we used to fit the data. We added a line at $1.3 \mathrm{keV}$ (roughly where the $\mathrm{Mg} \mathrm{K} \alpha$ line should be) to the model, fixed the energy and the width of the gaussian profile, and fitted for the intensity of the line. We found that $\chi^{2}$ dropped by more than 30 when the line was included. The best-fit equivalent width (derived from the SIS) was $40 \mathrm{eV}$. This extra Mg emission could be the result of nonequilibrium ionization effects or enhanced abundances, but the statistical precision of the $A S C A$ data is insufficient to allow us to give a definitive explanation at this time. Nevertheless, these clear indications of thermal emission, combined with our spatial study, allow us to conclude that we are seeing the two components predicted by the standard model of SNR evolution: soft shell-like emission (temperature $\simeq 0.8 \mathrm{keV}$ ) from the interaction of the shock wave with the interstellar medium and the hard nonthermal contribution arising from synchrotron emission of relativistic electrons in the magnetic field of the nebula surrounding the neutron star.

\subsection{Timing Analysis}

As emphasized in the previous section, the presence of a compact object in MSH 11-62 is unambiguous and so one might expect to detect the pulsed signal coming from the the 
neutron star itself. In fact, not every existing pulsar is expected to be detected; beaming effects, for example, may result in a cone of emission which does not intersect the Earth. This beaming effect presumably accounts for the relatively low number of SNRs in which a radio pulsar, undoubtedly associated with the remnant, has been discovered. In fact, of the 24 cataloged SNRs (Green 1996) that show some evidence for a nonthermal X-ray spectrum, only half have been associated with radio pulsars. In addition, a recent paper (Kaspi et al. 1996) presented the results of a search for radio pulsars in a sample of 40 southern Galactic SNRs. MSH 11-62 was part of the sample investigated but no pulsar was detected in the vicinity of the SNR. The limits on the radio flux density deduced from this unsuccessful search are shown on Fig. 5.

Because our primary emphasis was to acquire accurate spectral data, the observational configuration of our data set was not optimized for timing analysis. Moreover the statistics are low: after further restricting ourselves to the high-energy $(>4 \mathrm{keV})$, central portion of the remnant there were only $\sim 1230$ events collected at a time resolution of $125 \mathrm{~ms}$ and even fewer $(<300)$ in high-bit-rate mode (resolution $\sim 16 \mathrm{~ms}$ ). Both FFT and $Z_{\mathrm{n}}$ tests for the $125 \mathrm{~ms}$ resolution data found some possible periodicities (at about the $3 \sigma$ level), suggestive of a pulsed fraction of $\sim 10 \%$, but the results were not confirmed in the high-bit-rate data and remain unconvincing. If there was a pulsar and it was 10\%-pulsed, then we would expect to see a point source in the IPC image with an average count rate of $6.0_{-1.3}^{+2.4} \times 10^{-3}$

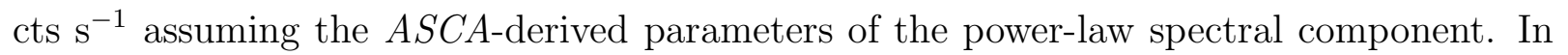
fact there is no central point source in the IPC image with a count rate this large, effectively ruling out a pulse fraction of $10 \%$ or greater.

\section{Discussion}




\subsection{Distance to MSH 11-62}

As is often the case in astronomy, the distance to MSH 11-62 is not well known. No measure of Hi absorption is available for this source and, thus, the method sometimes used in this situation correlates the remnant's surface brightness, $\Sigma$, (a distance independent quantity) to its diameter, $D$. This is the $\Sigma-D$ relation in which, for a sample of SNRs, one fits a power-law function to the variation of $\Sigma$ with $D$. This method has been applied to MSH 11-62 (Clark \& Caswell 1976; Milne 1979) and leads to a distance of 7-11 kpc, where the range comes from the sample of SNRs on which the relation is defined. The $\Sigma-D$ relation is, however, known to be unreliable, especially for centrally-brightened SNRs like MSH 11-62. A better estimate of the distance, for Crab-like SNRs, can be derived using the $S \theta^{2}-D$ relation (Weiler \& Panagia 1980). As opposed to the shell-like SNRs used in the $\Sigma-D$ relation, only plerions are used in this method that links the flux density $(S)$ and angular size $(\theta)$ to the distance $(D)$. Using this method, the distance to MSH 11-62 is found to be about $6 \mathrm{kpc}$. Yet another estimate of the distance to MSH 11-62 can be found using a $\Sigma-D$ relation by Reynolds \& Chevalier (1984) adjusted to model the Crab very well. This gives a small distance (between 1 and 6 kpc). Finally, Roger et al. (1986) suggest a distance to MSH 11-62 similar to that of two HII regions (G290.6+0.3 and G291.3-0.7) present in the vicinity of the SNR. This hypothesis leads to a distance of $3.5 \mathrm{kpc}$, in agreement with the bounds found in the Reynolds \& Chevalier model.

Another method to estimate the distance uses the correlation between column density (absorption along the line of sight) and distance (see Fig. 3 in Seward et al. 1972). For a column density of $6 \times 10^{21}$ atoms $\mathrm{cm}^{-2}$, this method gives an estimate of the distance between 2 and $6 \mathrm{kpc}$. One should note that this method makes use of an average density along the line of sight, an approximation which certainly introduces a considerable uncertainty in the distance estimate. As we discuss below, one interpretation of the X-ray results is suggestive of a larger distance than those mentioned previously. In our analysis, 
we have kept the distance dependence explicit and express all our results as a function of $D_{10}$ (where $D_{10}$ is the distance normalized to $10 \mathrm{kpc}$.)

\subsection{Dynamical Evolution of MSH 11-62}

From the size of the remnant, the observed emission measure, and the distance, one can estimate the density of the X-ray-emitting gas in MSH 11-62. The volume of the emission region defined by the total extent of the SNR (an angular radius of 6.4) assuming spherical symmetry is $V=7.9 \times 10^{59} \mathrm{fD}_{10}^{3} \mathrm{~cm}^{-3}$, where $f$ is the volume filling factor of the hot X-ray-emitting gas, which is less than unity. Combined with the observed emission measure determined from the spectral analysis (Table 1 ) and a ratio of $n_{e} / n_{\mathrm{H}}=1.17$, we deduce a hydrogen number density of $n_{\mathrm{H}}=\left(5.0_{-0.7}^{+1.1}\right) \times 10^{-2} D_{10}^{-1 / 2} \mathrm{f}^{-1 / 2} \mathrm{~cm}^{-3}$. This is the mean density of the shocked gas and is independent of the dynamical state of the remnant. We study the dynamical evolution of MSH 11-62 assuming two extreme values for the remnant's distance. The short distance, $3.5 \mathrm{kpc}$, is consistent with the distance determined by the X-ray column density, the HiI regions, and the Reynolds \& Chevalier $\Sigma-D$ relation.

For this distance, we find a mean density in the remnant of $n_{\mathrm{H}} \sim 8.5 \times 10^{-2} f^{-1 / 2} \mathrm{~cm}^{-3}$ and a mass of X-ray emitting plasma of $M_{X}=3.2 f^{1 / 2} M_{\odot}$ (assuming a helium abundance 0.085 times hydrogen by number). Given this small amount of mass, the remnant clearly cannot be in the Sedov-Taylor phase of evolution (Taylor 1950; Sedov 1959) and should rather be considerably younger. One limit on the age is set by assuming undecelerated expansion of the remnant at $5000 \mathrm{~km} \mathrm{~s}^{-1}$, which gives a free expansion age of $1300 \mathrm{yr}$. (The remnant is $6.5 \mathrm{pc}$ in radius at this distance.) A firm upper limit of $3500 \mathrm{yr}$ is provided by (erroneously!) assuming Sedov-Taylor expansion (see below). If this scenario is correct, then it is perhaps surprising that the thermal emission from MSH 11-62 does not show stronger evidence for enhanced metal abundances, which might be expected from the presence of reverse-shock heated ejecta. The low density of the ambient medium would, however, mitigate against 
a strong reverse shock. A similar absence of an ejecta component is also observed for the Crab Nebula and 3C58, both of which reside in low density environments. Finally, we note that the mean thermal pressure inside the SNR is $P=n k T \sim 2.5 \times 10^{-10} f^{-1 / 2}$ dyne $\mathrm{cm}^{-2}$, where $n$ is the total number density of particles (electrons and ions) and the temperature, $k T$, is the same for all species.

In view of the large uncertainties that plague our estimates of the distance to MSH 11-62, we have examined the possibility that the remnant is located at a much larger distance, $10 \mathrm{kpc}$. In this case the hot gas mass is considerable, $M_{X}=44 f^{1 / 2} M_{\odot}$, and so it is reasonable to assume that the remnant is in the Sedov-Taylor or adiabatic phase of evolution. The self-similar solutions to the hydrodynamical evolution of adiabatic-phase SNRs provide a numerical relationship between the age of the remnant and the size and temperature of the shock wave. The temperature determined by our spectral analysis (§2.2), $\langle T\rangle$, is the emission-measure-weighted average electron temperature, which is proportional to the shock temperature, $\langle T\rangle=1.27 T_{S}$ (assuming, as above, that electrons and ions have the same temperature). The Sedov age relationship is $t \approx 480 \mathrm{yrs}(R / 1 \mathrm{pc})(\langle T\rangle / 1 \mathrm{keV})^{-1 / 2}$, and so the measured angular size and temperature of MSH 11-62 imply an age of $\simeq 10^{4} D_{10}$ yr. We estimate the preshock Hydrogen number density $n_{0}$ by integrating the interior radial density variation from the Sedov similarity solution and then equating it to the observed emission measure. This gives a preshock density of $n_{0}=(3.4 \pm 0.7) \times 10^{-2} D_{10}^{-1 / 2} \mathrm{~cm}^{-3}$. With the preceding numerical values we estimate the supernova explosion energy from the Sedov relations to be $E=(0.25 \pm 0.05) \times 10^{51} D_{10}^{5 / 2}$ ergs. The central pressure $P_{0}$ in the Sedov similarity solution is related to the pressure at the shock front as $P_{0}=0.31 P_{S}$. Evaluting using the best fit numerical quantities we find $P_{0} \sim 9.6 \times 10^{-11}$ dyne $\mathrm{cm}^{-2}$. 


\subsection{MSH 11-62 and the EGRET Source 2EG J1103-6106: A Doubtful Association}

The Energetic Gamma-Ray Experiment Telescope (EGRET) on board the Gamma Ray Observatory detected a source in the general vicinity of MSH 11-62 with an integrated flux from $100 \mathrm{MeV}$ to $30 \mathrm{GeV}$ of $(5.6 \pm 0.9) \times 10^{-7}$ photons $\mathrm{cm}^{-2} \mathrm{~s}^{-1}$. The second EGRET catalog (Thompson et al. 1995) lists the source as 2EG J1103-6106 and gives a position of $11^{\mathrm{h}} 03^{\mathrm{m}} 48^{\mathrm{s}},-61^{\circ} 06^{\prime}(\mathrm{J} 2000)$ with an error ellipse of size $49^{\prime} \times 32^{\prime}$ that is only marginally consistent with the position of MSH 11-62. For this source, the EGRET team (Merck et al. 1996) reports a spectral index of $-2.3 \pm 0.2$ (marginally consistent with the X-ray spectral index reported here). Fig. 5 shows the EGRET spectrum of 2EG J1103-6106 in comparison to data on MSH 11-62 from radio to X-ray wavebands. The $\gamma$-ray data are inconsistent with a simple extrapolation of the X-ray power-law spectrum to higher energies. Note that the total number of photons detected by EGRET is small, only 312, and that the position is uncertain by more than $30^{\prime}$. Moreover, a recent paper (Kaspi et al. 1997) reported the discovery of a pulsar, possibly associated with the nearby SNR MSH 11-61A, at a position that agrees with the error box of 2EG J1103-6106. These authors conclude that the $\gamma$-ray source and this pulsar were likely to be associated. Our spectral results are consistent with this interpretation (i.e., that the EGRET source and MSH 11-62 are not associated) and so we exclude the EGRET $\gamma$-ray source from further discussion.

\subsection{Empirical Models for the Synchrotron Nebula}

We have argued above that the nonthermal emission is coming from the synchrotron nebula surrounding a spinning neutron star, or pulsar, created in the supernova explosion. From the empirical relationship between a pulsar's spin-down energy $\dot{E}$ and the power-law X-ray luminosity of the nebula $L_{X}$ (Seward \& Wang 1988), which is $(2.5 \pm 0.3) \times 10^{34} D_{10}^{2}$

$\operatorname{ergs~s}^{-1}(0.2-4.0 \mathrm{keV})$, we infer $\dot{E} \sim(1.0 \pm 0.9) \times 10^{37} D_{10}^{1.44} \mathrm{ergs} \mathrm{s}^{-1}$ for the putative pulsar 
in MSH 11-62.

In figure 5 we plot the available radio spectral data (Roger et al. 1986) and the results of the power-law fits for the X-ray synchrotron nebula. For the following discussion the points on the figure indicating the upper limits to the flux of the radio pulsar (downward-pointing arrows on the left side) and the EGRET $\gamma$-ray source (plus symbols on the right side) should be ignored. We can use the radio spectral data, the well-established theory for synchrotron radiation, and a minimum total energy (in fields and particles) assumption to estimate the magnetic field strength in the nebula. For a power-law distribution of electrons, one can write (Ginzburg \& Syrovatskii, 1965, eqn. 5.16):

$$
B_{1}=\left[48 \kappa_{\mathrm{m}} \kappa_{\mathrm{r}} A(\gamma, \nu) \frac{F_{\nu}}{D \theta^{3}}\right]^{2 / 7}
$$

where $\kappa_{\mathrm{m}}$ is the constant of proportionality between the energy in magnetic field and relativistic particles (minimum total energy is obtained for $\kappa_{\mathrm{m}}=3 / 4$, which is the value we use) and $\kappa_{\mathrm{r}}$ is the proportionality constant between the energy in relativistic electrons and the energy in cosmic rays of all species. If the protons and electrons are assumed to have the same energy density throughout the source, then we have $\kappa_{\mathrm{r}}=2$ which is the value adopted in the following computation. $A(\gamma, \nu)$ is a numerical coefficient (which is calculated from the physics of synchrotron radiation) that depends on the slope of the electron energy spectrum $(\gamma)$ and the frequency range over which the power-law distribution is valid. In the radio band $\gamma=2 \alpha+1$, where $\alpha$ is the radio spectral index (defined as $\left.F_{\nu} \sim \nu^{-\alpha}\right)$. The quantities $D$ and $\theta$ are respectively the distance to the object and its angular diameter. Note that the frequency dependence of the quantity $A(\gamma, \nu)$ is cancelled by the corresponding $\nu$ dependence of the spectral flux density of the source, $F_{\nu}$. One can write (explicitly including the distance dependence):

$$
B_{1}=46.9 D_{10}^{-2 / 7}\left(\theta_{\mathrm{P}} / 2^{\prime} .5\right)^{-6 / 7}\left[\kappa_{\mathrm{m}} \kappa_{\mathrm{r}}\right]^{2 / 7} \mu \mathrm{G}
$$

For the size of the radio plerion we derived an average angular radius $\theta_{\mathrm{P}}=2.5$ from the MOST radio image as discussed above. Table 2 summarizes the values of $B_{1}$ found for the 
two different distances considered.

The broadband spectrum of the nebula in MSH 11-62 shows a clear spectral break somewhere between the radio and the X-ray band. The break frequency $\nu_{\mathrm{B}}$, which is where the extrapolated X-ray and radio power-law spectra intersect, lies in the range 8-200 GHz. The lower limit corresponds to not violating any of the radio measurements, while the upper limit is derived from the largest allowed slope of the X-ray power-law spectrum. Assuming that $\nu_{\mathrm{B}}$ represents the frequency at which synchrotron losses begin to dominate, we can obtain another estimate of the nebular magnetic field. In this case, we find

$$
B_{2}=740\left(\nu_{\mathrm{B}} / 35 \mathrm{GHz}\right)^{-1 / 3}\left(t / 10^{4} \mathrm{yrs}\right)^{-2 / 3} \mu \mathrm{G}
$$

which comes directly from Ginzburg \& Syrovatskii (1965, eqn. 5.36). Here $t$ is the age of the nebula, for which we use the values derived in our study of the thermal emission (tabulated in Table 2). This estimate of the magnetic field strength has a large uncertainty because of the large range in break frequencies. Furthermore, this method is to be considered with caution. The break frequency deduced from extrapolating the X-ray and radio spectra is not a well defined physical quantity. In fact, there are other remnants [e.g. 3C58 (Green \& Scheuer 1992)] for which no simple extrapolation from X-ray to radio spectra is possible, suggesting other evolutionary effects for the central pulsar. In such cases, computation of the magnetic field with this method is no longer valid.

Yet a third method to compute the magnetic field supposes pressure equilibrium between the hot thermal gas in the center of the SNR and the synchrotron nebula so that $B_{3}=\sqrt{8 \pi P_{0}}$. We obtained estimates of the pressure in the hot gas in $\S 3.2$ for both assumed distances to the remnant (values are shown in Table 2) which result in values for $B_{3}$ of 79 $\mu \mathrm{G}$ and $50 \mu \mathrm{G}$. These values are potentially rather uncertain as well. The most significant uncertainty arises from our assumption during the analysis of the thermal emission that the electron and ion temperatures in the remnant were equal. It is possible and perhaps likely that the electrons have significantly lower temperatures than the ions because the timescale 
for exchange of energy between these different species is quite long if only Coloumb collisions operate. In fact the protons could have 10 to 100 times the temperature of the electrons and that would increase our magnetic field estimates by factors of 3 to 10 .

\subsection{Interpretation}

The two different distance regimes discussed above lead to distinctly different evolutionary stages for MSH 11-62. At a distance of $3.5 \mathrm{kpc}$, the remnant is dynamically young, having swept up only a small amount of mass. The ambient density $n_{0} \sim 0.04 \mathrm{~cm}^{-3}$ (assuming a volume filling factor of $f=1 / 4$ and the strong shock compression factor of 4) is quite low, possibly indicating evolution in a wind-blown cavity created by the massive precursor star implied by the presence of the compact remnant. The luminosity of the synchrotron nebula is $L_{\text {synch }}=3.1 \times 10^{33} \mathrm{erg} \mathrm{s}^{-1}$, suggesting a central pulsar with a spin-down energy loss rate of $\dot{E}=2.5 \times 10^{36} \mathrm{erg} \mathrm{s}^{-1}$. For a surface magnetic field of $B_{s}=10^{12}-10^{13} \mathrm{G}$ and a braking index $\nu=2.5-3$, this implies an initial spin period in the range $P_{0} \sim 45-130 \mathrm{~ms}$ with a current-epoch period in the range $P_{0} \sim 46-160 \mathrm{~ms}$. The magnetic pressure associated with the synchrotron nebula is $B_{n e b} \sim 70 \mu \mathrm{G}$ and is in close equilibrium with the thermal pressure of the SNR interior.

For a distance as large as $10 \mathrm{kpc}$, MSH 11-62 would be in the Taylor-Sedov phase of evolution. The swept-up mass $\approx 30 M_{\odot}$ is sufficiently large to explain the lack of a significant ejecta component to the thermal emission, and the associated explosion energy $E_{51} \approx 0.25$ is rather typical of SNRs. The remnant is then $\sim 10^{4} \mathrm{yr}$ old and harbors a synchrotron nebula whose luminosity is $L_{\text {synch }}=2.5 \times 10^{34} \mathrm{erg} \mathrm{s}^{-1}$. The associated spin-down power is $\dot{E}=10^{37} \mathrm{erg} \mathrm{s}^{-1}$ and the neutron star parameters above yield an initial spin period range of $29-46 \mathrm{~ms}$ with a current-epoch period in the range of $31-100 \mathrm{~ms}$. (We note that for some values of $P_{0}$ in the above range, $P$ and $B_{s}$ are double-valued, corresponding to solutions for $P \approx P_{0}$ and $P$ significantly larger than $P_{0}$.) These parameters are similar to other young 
pulsars (e.g. PSR B1509-58 in MSH 15-52, or PSR B1800-21 in G8.7-0.1) making this scenario plausible. However, the ambient density $n_{0} \sim 3.4 \times 10^{-2} \mathrm{~cm}^{-3}$ is rather small. One might again invoke a pre-SN wind-blown cavity as an explanation, but in this case the size of the nebula would need to be considerable $\sim 20$ pc in radius. Local variations in density by factors as large as 5 or more are observed in the direction of MSH 11-62 (Burton \& te Lintel Hekkert 1986), but the integrated column density is also problematic. The total column density integrated through the entire galaxy in the direction toward MSH 11-62 is $N_{H}=1.5 \times 10^{22} \mathrm{~cm}^{-2}$ (Dickey \& Lockman 1990) while that for $D>10 \mathrm{kpc}$ is only $\sim 3 \times 10^{21} \mathrm{~cm}^{-2}$ (obtained by rough integration of the Hi gas density maps of the outer Galaxy from Burton \& te Lintel Hekkert). It would thus appear difficult to place the remnant at $10 \mathrm{kpc}$ given the column density derived from the spectral fits. We note, however, that the Hi values are averaged over a grid size larger than the size scale of the remnant, so that fluctuations corresponding to the observed X-ray column density over a distance of $10 \mathrm{kpc}$ cannot be completely ruled out.

Hi absorption measurements for the SNR would lead to an accurate determination of the distance which would resolve the ambiguity in determination of the evolutionary state of the remnant. If MSH 11-62 is indeed older and more distant, then one might expect the blast wave to have encountered a number of clouds in which the shocks have become complete. Thus, optical observation of $\mathrm{H}_{\alpha}$ and [OIII] or [SII] would be of interest in attempting to differentiate between the two scenarios.

\section{Summary}

We have presented the results of $A S C A$ X-ray spectral and spatial studies of the SNR MSH 11-62. This remnant is now clearly identified as a composite SNR with an unresolved central synchrotron-emitting component powered by a compact object and an extended thermal component of ISM plasma shock-heated by the SN blast wave. No pulsed signal 
from the unresolved central component is detected in the X-ray band, consistent with the failure of previous radio searches to detect a radio pulsar. We find that we cannot confidently determine the evolutionary state of the remnant; the main problem is the lack of a good distance estimate. If the remnant is nearby $(3.5 \mathrm{kpc})$, it is young $(<3500 \mathrm{yr})$, has not reached the Sedov phase, and is evolving in a very low density environment. If the remnant is further away, say $10 \mathrm{kpc}$, then it would be a middle-aged remnant in its Sedov phase of evolution, with an initial explosion energy of $\sim 2 \times 10^{50}$ ergs. For either distance we can find a set of parameters that can plausibly describe the central pulsar now powering the synchrotron nebula. Likewise we can develop an acceptable description for the synchrotron nebula that is consistent with those of other known synchrotron nebulae for which the powering neutron star has been detected by radio or X-ray timing searches. Our results confirm the power of direct imaging and spectral analysis of X-ray data for detecting compact objects inaccessible to pulse searches. It does not fall prey to beaming effects and this technique should be used more extensively to survey all the cataloged SNRs that have, as does MSH 11-62, a flat and highly polarized radio spectrum.

David Helfand and Philip Kaaret carefully read drafts of this paper and we thank them for their many comments that improved it. We thank Bryan Gaensler for providing the MOST data in electronic form. This research was supported in part by NASA under grants NAG 5-3486 and contract NAS 8-39073. JPH acknowledges support from NASA Grants NAG 5-4871 and NAG 5-4794. 


\section{REFERENCES}

Anders, E., \& Grevesse, N. 1989, Geochimica et Cosmochimica Acta, 53, 197

Blanton, E. L., \& Helfand, D. J. 1996, ApJ, 470, 961

Burton, W. B., \& te Lintel Hekkert, P. 1986, A \& A Suppl, 427, 65

Clark, D. H., \& Caswell, J. L. 1976, MNRAS, 174, 267

Dickey, J. M., \& Lockman, F. J. 1990, ARA \& A, 28, 215

Ginzburg, V. L. \& Syrovatskii, S. I. 1965, ARA \& A, 3, 297

Green, D. A. 1996, 'A Catalogue of Galactic Supernova Remnants (August version)'

Green, D. A. \& Scheuer, P. A. G. 1992, MNRAS, 258, 833

Harrus, I. M., Hughes, J. P., \& Helfand, D. J. 1996, ApJ, 464, L161

Kaspi, V. M. et al. 1997, ApJ, 485, 820

Kaspi, V. M., Manchester, R. N., Johnston, S., Lyne,A. G., \& D’Amico, N. 1996, A J, 111, 2028

Merck, M. et al. 1996, A\&A Suppl, 120, 465

Milne, D. K. 1979, Aust. J. Phys., 32, 83

Morrison, R. \& McCammon, D. 1983, ApJ, 270, 119

Raymond, J. C. \& Smith, B. W. 1977, ApJS, 35, 419

Reynolds, S. P. \& Chevalier, R. A. 1984, ApJ, 278, 630

Roger, R. et al. 1986, MNRAS, 219, 815 
Sedov, L. I. 1959, Similarity and dimensional methods in mechanics, (New York: Academic Press)

Seward, F. D. \& Wang, Z. R. 1988, ApJ, 332, 199

Seward, F. D. et al. 1972, ApJ, 178, 131

Slane, P. O., Seward, F. D., Bandiera, R., Torii, K., \& Tsunemi, H. 1997, ApJ, 485, 221

Tamura, K., Kawai, N., Yoshida, A., \& Brinkmann, W. 1996, PASJ, 48, L33

Taylor, G. I. 1950, Proc Royal Soc London, 201, 159

Thompson, D. J. et al. 1995, ApJS, 101, 259

Vasisht, G., Aoki, T., Dotani, T., Kulkarni, S. R., \& Nagase, N. 1996, ApJ, 456, L59

Weiler, K. W. \& Panagia, N. 1980, A\&A, 90, 269

Whiteoak, J. B. Z. \& Green, A. J. 1996, A \& A Suppl. Ser., 118, 329

Wilson, A.S. 1986, ApJ, 302, 718 
Fig. 1.- $A S C A$ X-ray images of the SNR MSH 11-62 in three X-ray bands (top: 0.5$2.0 \mathrm{keV}$; middle: $2.0-4.0 \mathrm{keV}$; bottom: $\gtrsim 4.0 \mathrm{keV}$ ) from the GIS (left) and the SIS (right). Contour values are linearly spaced from $30 \%$ to $90 \%$ of the peak surface brightness in each map. Peak/background values are top: 1.4/0.084; middle: 1.3/0.042; bottom: 0.81/0.053 for the GIS and top: 2.5/0.145; middle: 1.5/0.042; bottom: 0.71/0.053 for the SIS, where all values are quoted in units of $10^{-3}$ counts s$~^{-1} \operatorname{arcmin}^{-2}$.

Fig. 2.- Same as Fig. 1 with the radio contours from the MOST survey superimposed. The radio contours are linearly spaced from a minimum value of $0.027 \mathrm{Jy}$ to a maximum of 0.27 Jy.

Fig. 3.- Variation of the X-ray hardness ratio as a function of radius for MSH 11-62. Both GIS (with detectors 2 and 3 combined) and SIS (with detectors 0 and 1 combined) profiles are shown.

Fig. 4.- GIS and SIS spectra of MSH 11-62 extracted from circular regions centered on the remnant. There were 9709 GIS events within a circle of radius $5^{\prime}$ and 8908 SIS events within a circle of radius $4^{\prime}$. The solid curves in the top panels show the joint best-fit Raymond \& Smith thermal plasma plus power-law models. The insert figures show only the thermalcomponent; above $2.0 \mathrm{keV}$, its contribution is an order of magnitude less than the power-law emission. The bottom panels plot the data/model residuals. There appears to be excess emission near $1.3 \mathrm{keV}$, which can be modeled well as a $\mathrm{K} \alpha$ line of helium-like magnesium (see text). 
Fig. 5.- Broadband plot of the spectral flux density (in Jy) vs. frequency for MSH 11-62. The radio points correspond to frequencies of $0.408 \mathrm{GHz}, 1 \mathrm{GHz}, 5 \mathrm{GHz}$, and $8.4 \mathrm{GHz}$ (Roger et al. 1986) and the line through them denotes the best radio spectral index (dotted when extrapolated beyond the frequency range of the measurements). Also indicated (as downward-pointing arrows near the left side of the figure) are the upper limits on a pulsed component to the radio flux (Kaspi et al. 1996). In the X-ray band $\left(\sim 10^{17} \mathrm{~Hz}\right)$, the $A S C A$ spectral results for the power-law component are shown as two curves bounding the allowed range of X-ray spectral indices. Extrapolations of the X-ray spectra beyond the $A S C A$ band (0.5-10 keV) are shown dashed. The $\gamma$-ray spectrum of the EGRET source 2EG J1103-6106 (Merck et al. 1996) is shown, although the association of this source with MSH 11-62 is unlikely (see text). 
Table 1

Spectral Analysis of Power Law plus Thermal a Plasma Model

\begin{tabular}{|c|c|}
\hline Parameter & Fit results ${ }^{b}$ \\
\hline$N_{\mathrm{H}}\left(\right.$ atoms $\left.\mathrm{cm}^{-2}\right)$ & $(0.6 \pm 0.1) \times 10^{22}$ \\
\hline$\alpha_{\mathrm{p}}$ & $2.0_{-0.3}^{+0.1}$ \\
\hline$F_{1 \mathrm{keV}}\left(\right.$ photons $\left.\mathrm{cm}^{-2} \mathrm{~s}^{-1} \mathrm{keV}^{-1}\right)$ & $(1.26 \pm 0.13) \times 10^{-3}$ \\
\hline$k T(\mathrm{keV})$ & $0.80 \pm 0.05$ \\
\hline $\mathrm{EM}^{\mathrm{c}}\left(\mathrm{cm}^{-5}\right)$ & $\left(1.9_{-0.5}^{+1.0}\right) \times 10^{11}$ \\
\hline$\chi^{2} / \nu$ & $671.9 / 336$ \\
\hline \multicolumn{2}{|c|}{ a Cosmic abundances (Anders \& Grevesse 1989) } \\
\hline b Single-parameter $1 \sigma$ errors & \\
\hline${ }^{\mathrm{c}}$ Emission measure $\left(\frac{n_{\mathrm{H}} n_{e} V}{4 \pi D^{2}}\right)$ & \\
\hline
\end{tabular}


Table 2

Parameters derived for MSH 11-62

\begin{tabular}{lcc}
\hline \hline & \multicolumn{2}{c}{ Distance $(\mathrm{kpc})$} \\
\cline { 2 - 3 } Parameter & 3.5 & 10 \\
\hline$R_{\mathrm{S}}{ }^{\mathrm{a}}(\mathrm{pc})$ & 6.1 & 18.6 \\
Age $(\mathrm{yr})$ & $\sim 2500$ & $\sim 10^{4}$ \\
$R_{\mathrm{P}}{ }^{\mathrm{b}}(\mathrm{pc})$ & 2.5 & 7.3 \\
$P_{0}{ }^{\mathrm{c}}\left(10^{-10}\right.$ dyne $\left.\mathrm{cm}^{-2}\right)$ & $2.5 f^{-1 / 2}$ & 1.0 \\
$B_{1}(\mu \mathrm{G})$ & 72 & 53 \\
$B_{2}{ }^{\mathrm{d}}(\mu \mathrm{G})$ & $160-500$ & $400-1200$ \\
$B_{3}{ }^{\mathrm{c}}(\mu \mathrm{G})$ & $79 f^{-1 / 4}$ & 50 \\
\hline
\end{tabular}

${ }^{a}$ Angular size of shock radius is 6.4

b Angular size of radio plerion is 2.5

${ }^{\mathrm{c}} f$ is the volume filling factor

${ }^{\mathrm{d}}$ Range quoted corresponds to break frequencies between 8-200 GHz 

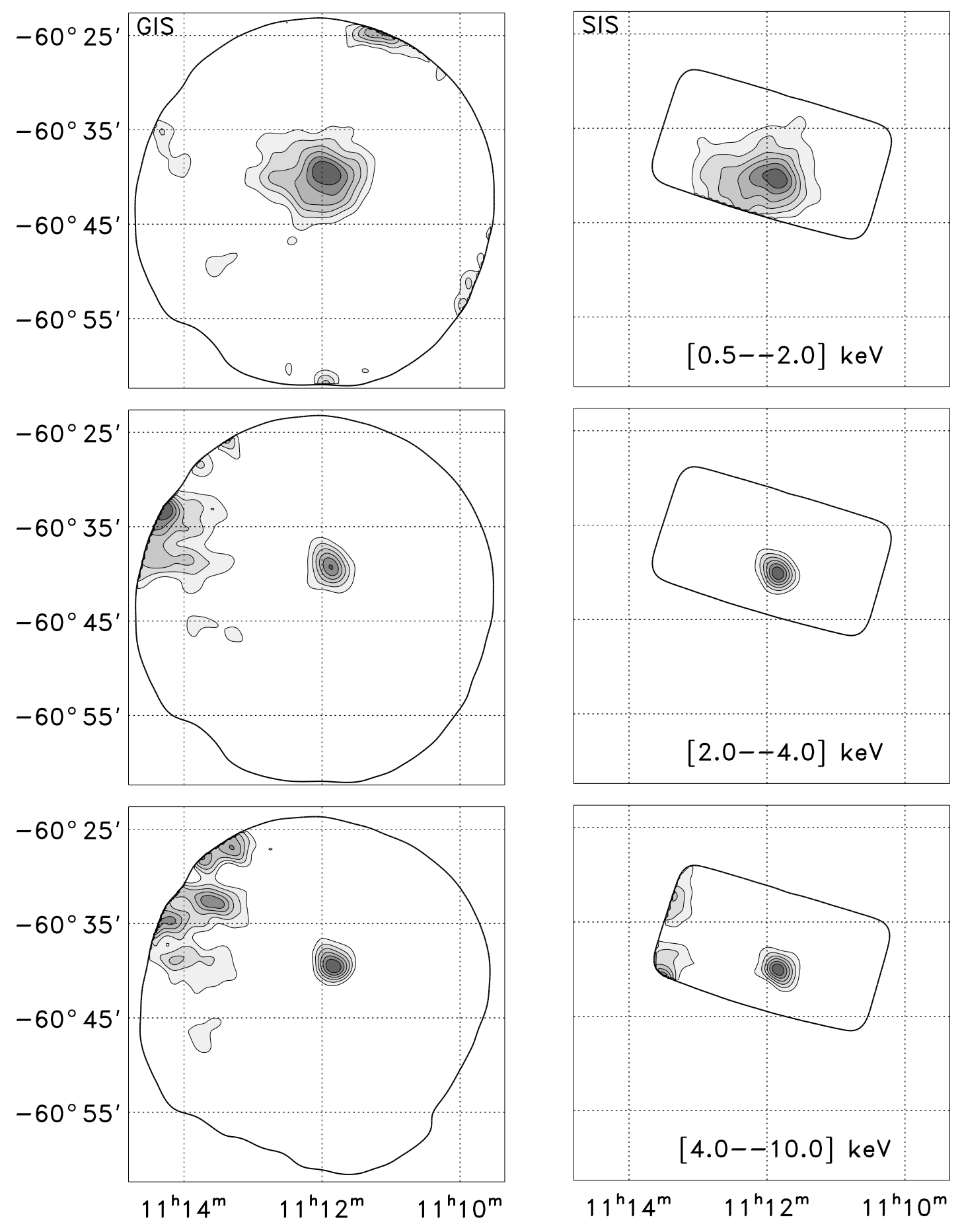

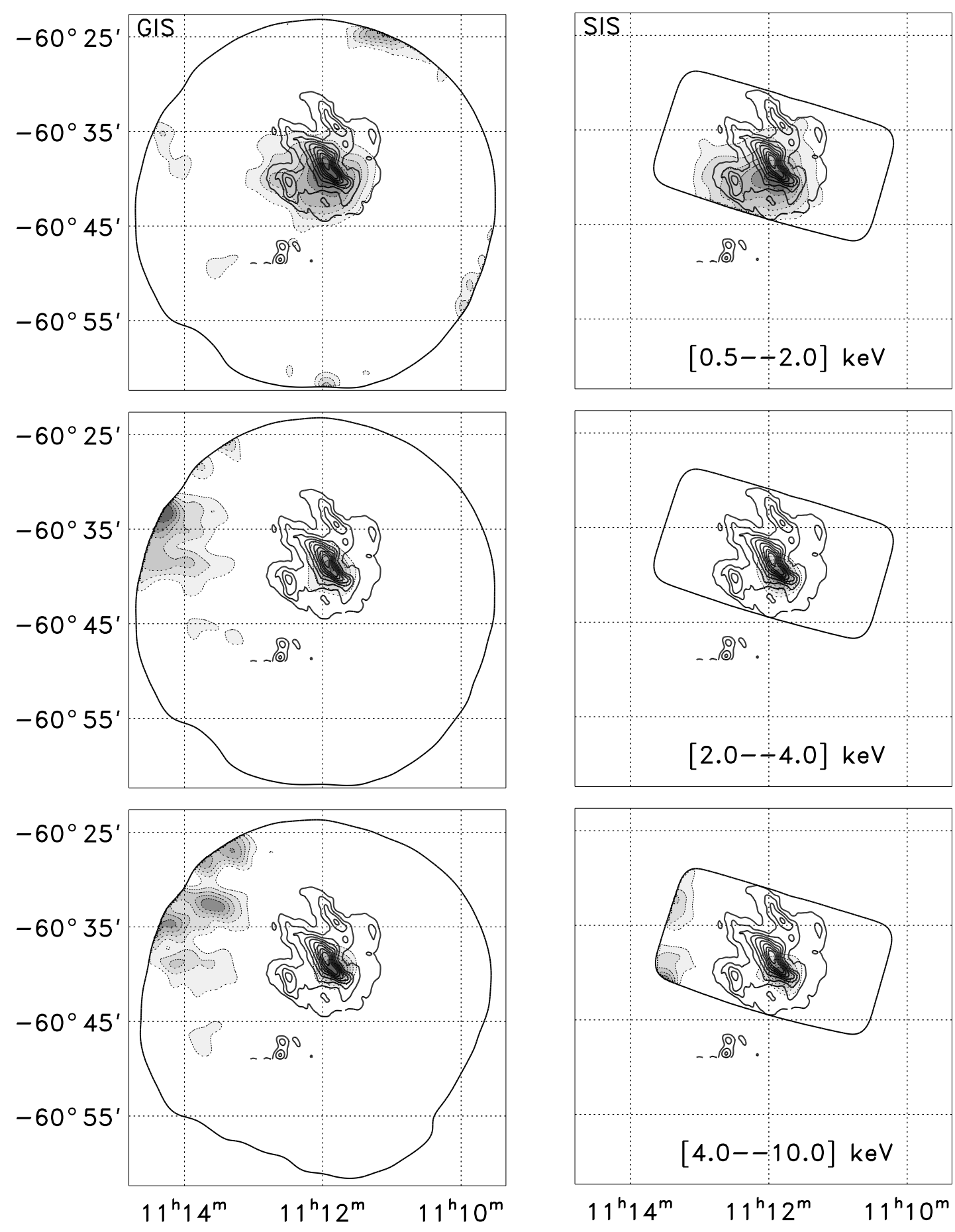


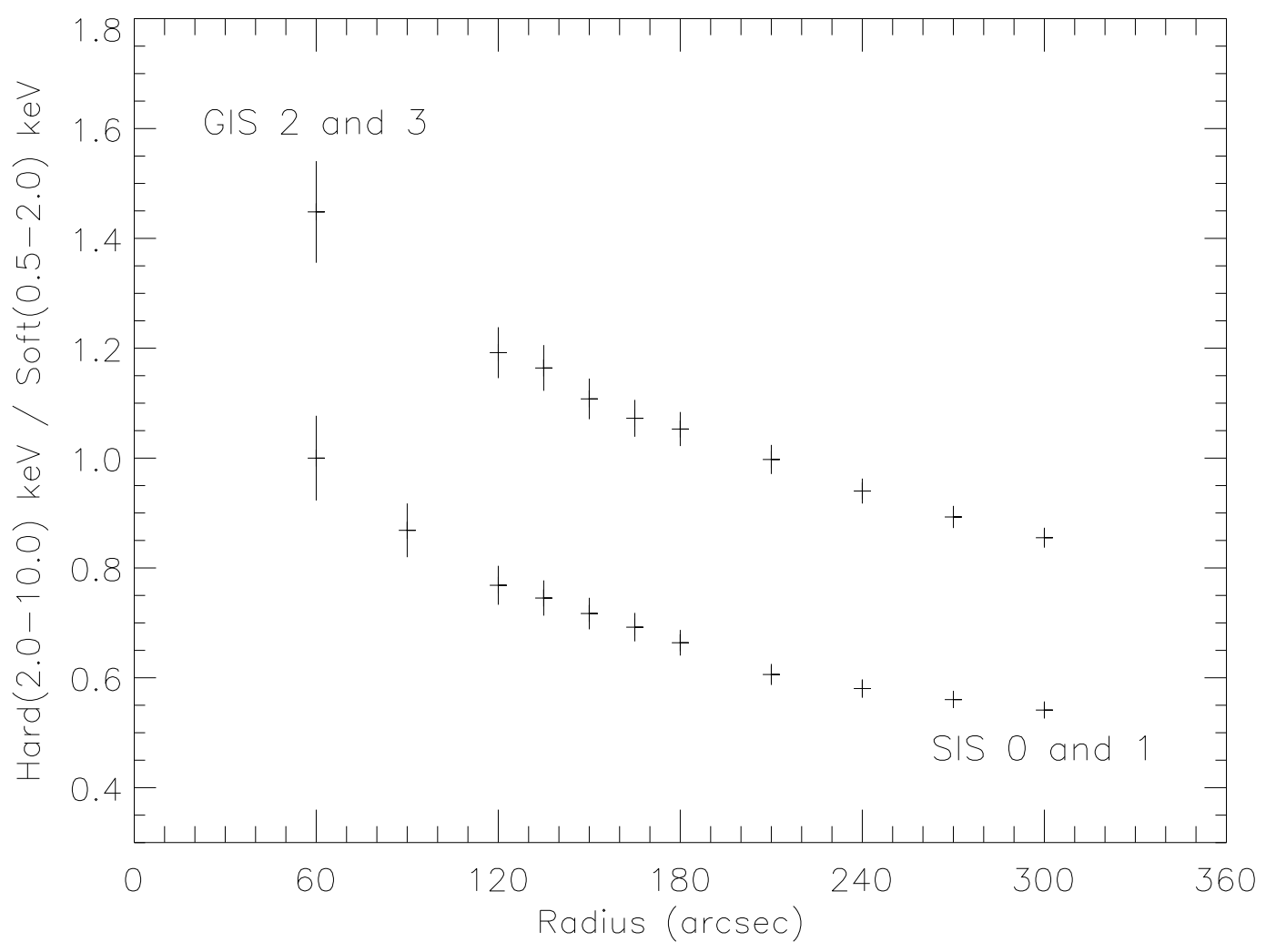



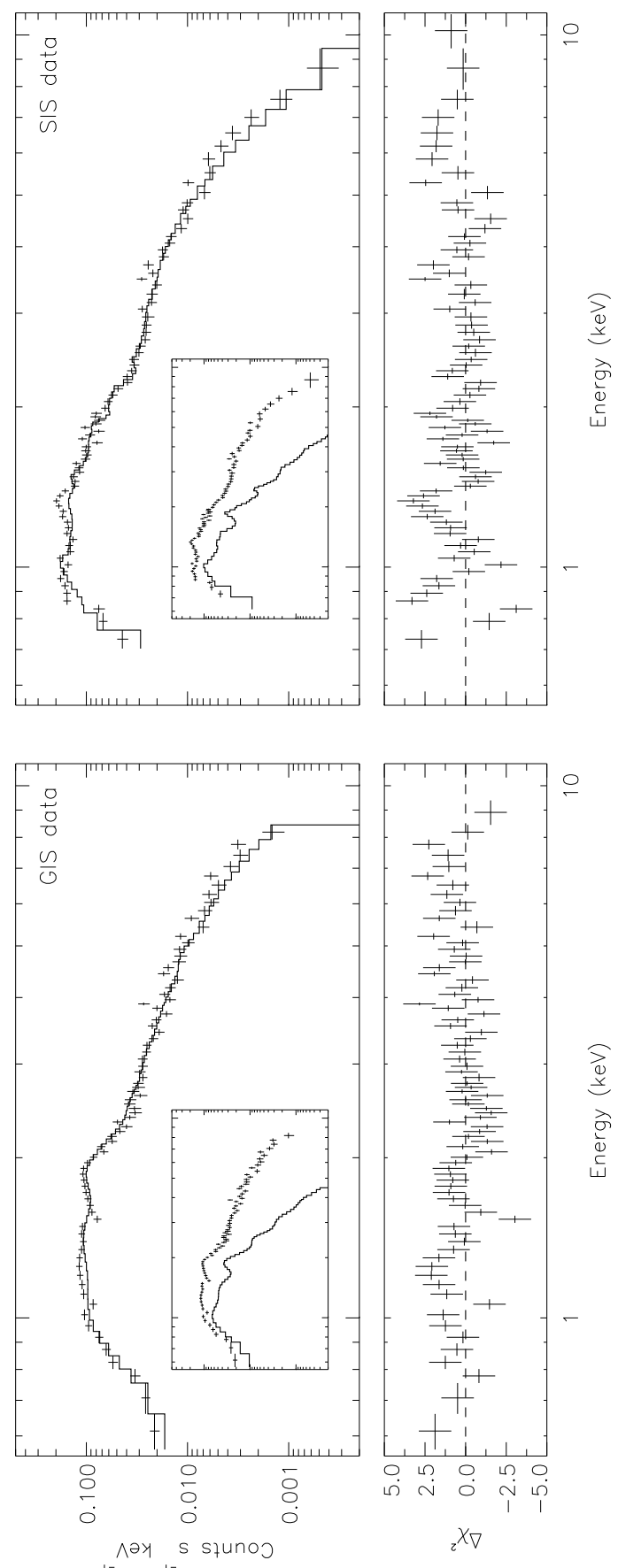


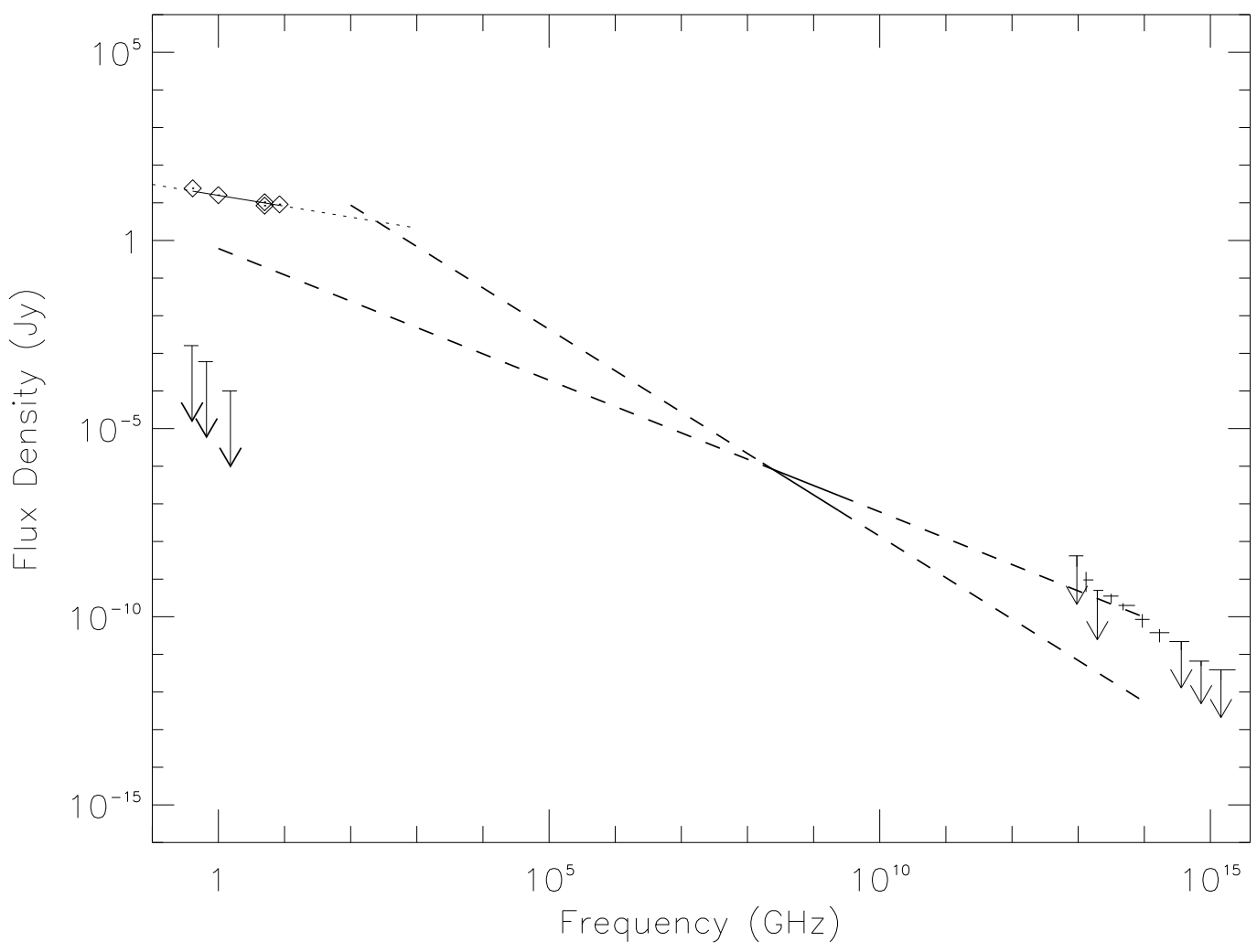

